\title{
The Pattern of Day Case (Ambulatory) Percutaneous (PCNL): A Descriptive Retrospective Study from a Tertiary Care Hospital
}

\author{
Rehan Fareed ${ }^{1,{ }^{*}}$ and Huma Shamim ${ }^{1}$ \\ ${ }^{1}$ General Surgery Department, Shri Ram Murti Smarak Institute of Medical Sciences, Bareilly, India \\ "Corresponding author: General Surgery Department, Shri Ram Murti Smarak Institute of Medical Sciences, Bareilly, India. Email: rehan.fareed@gmail.com
}

Received 2020 April 25; Revised 2020 November 07; Accepted 2021 February 07.

\begin{abstract}
Background: Percutaneous nephrolithotomy (PCNL) has experienced remarkable development and alteration since it was first described in 1976 by Fernstorm et al. It has also experienced miniaturization of equipment, improvement in operative systems, and refining renal access methods leading to the achievement of maximum clearance of stone while causing minimal morbidity. For example, in endourological practice, when the patient is subjected to PCNL, he traditionally needs programmed inpatient admission, as part of their recovery, it is applicable as an outpatient method in properly selected cases.

Objectives: We aimed at evaluating the safety and applicability of the outpatient PCNL procedure.

Methods: This retrospective study was done on 210 cases of tubeless PCNL performed by a single urologist at our institute from January 2016 to January 2019. Patients' mean age (134 males and 76 females) was $57 \pm 11.8$ years, and 7 patients aged 8 - 12 years. There were 71 pelvic or calyceal solitary stones, 62 non-complete staghorn stones, 17 ureteral stones, 32 renal + ureteric stones (simultaneous renal and ureteral stones), and 28 complete staghorn stones. The average stone size was $3.5 \pm 2.8$ (range: 0.7 to 11.8 $\mathrm{cm})$.

Results: The mean operation duration was $85.0 \pm 29.4 \mathrm{~min}$, and the mean hospital stay was $21.7 \pm 3.4 \mathrm{~h}$. Out of 210 patients, 6 patients had longer stay due to high-grade fever and 3 patients due to severe pain, and also 7 patients refused discharge due to personal and social reasons. Our ambulatory PCNL rate was $97 \%$ ( 194 out of 210). Within 72 h, 5 patients were readmitted due to high-grade fever, 3 patients due to haematuria, and 4 patients due to pain and dysuria, and all patients were discharged 2 - 4 days after conservative treatment. Thus, the readmission rate was $6.18 \%$ (12 cases were readmitted out of 194 cases). Patients showed a blood transfusion rate of $1.4 \%$. Also, 19 cases (9.02\%) were found with post-operative fever, and no urosepsis was reported. No pulmonary complications and mortality were noted. No re-exploration was done, and no major leak was noted. The angio-embolization rate was $0.59 \%$. We did not use HEMO-SEAL technology, cautery, or suture in the tracks.
\end{abstract}

Conclusions: In conclusion, the outpatient PCNL procedure is an applicable and feasible procedure under selected criteria; however, more investigations using a larger sample size are needed.

Keywords: Urolithiasis, PCNL

\section{Background}

Percutaneous nephrolithotomy (PCNL) has experienced remarkable development and alteration since it was first described in 1976 by Fernstorm et al. (1-3). It has also experienced miniaturization of equipment, improvement in operative systems, and refining renal access methods $(4,5)$ leading to the achievement of maximum clearance of stone while causing minimal morbidity. For example, in endourological practice, when the patient is subjected to PCNL, he traditionally needs programmed inpatient admission as part of his recovery, and it is applicable as an outpatient method in properly eligible cases. Ambulatory PCNL is regarded as cases being discharged the same day or 24 hours post-operatively and is also considered as a day case or outpatient PCNL. Alternative surgeries, like ureteroscopy, are currently commonly carried out on such a basis (6).

\section{Objectives}

We aimed at describing the pattern of evaluating the safety and applicability of an outpatient PCNL procedure.

\section{Methods}

This retrospective study was done on 210 cases of tubeless PCNL from January 2016 to January 2019 performed by 
a single urologist at our institute.

\subsection{Inclusion Criteria}

All types of kidney stones with upper and middle ureteric stones, normal renal function, normal contralateral kidney, and ASA grade 2.

\subsection{Exclusion Criteria}

Pyonephrosis, non-visualized kidney on intravenous pyelogram (IVP), radiolucent stone, residual stones (detected by real-time fluoroscopy), and those needed re-look PCNL.

All PCNL procedures were performed by a single urologist with standard operative methods under spinal or combined spinal-epidural (CSE) anesthesia. Different anesthetics were involved from the institute list. Through cystoscopy, the open-ended ureteral catheter was kept in the lithotomy position. If a stone present in the middle or upper ureter, then through ureteroscopy, the stone is pushed into the kidney with or without fragmentation. Renal access tract was obtained with Bull's eye technique in the prone position. Tract size varied from $26 \mathrm{~F}$ to $30 \mathrm{~F}$ and single tract to four tracts depending on the stone burden and renal anatomy. We applied a pneumatic lithotripter for stone fragmentation. After stone clearance, a $5 \mathrm{~F}$ double J stent was put in an antegrade manner to drain urine after surgery. All PCNL procedures were tubeless without percutaneous nephrostomy tube (PNT) was kept as it is our routine practice. Amplatz was removed, and 5-min hand compression was applied on the track site. No cautery or hemostatic gel or foam was used, and no suture was applied. A Foleys catheter was located in the body to drain urine, and the removal of the catheter was done the next morning. Patients' age, stone characteristic, and size, duration of operation, post-operative hospital stay, urinary tract infection, transfusion level, as well as other complications were noted and evaluated through retrospective chart review. Stone size measurement was done on KUB plain film through the longest diameter. The duration of operation was determined from cystoscopy to remove Ampaltz sheath. Stone free was considered as the total elimination of the stone as per fluoroscopy at the operation table. The temperature of above $101 \mathrm{~F}$ after operation or readmission due to that was defined as post-operative fever. Sepsis was regarded as a suspicion of infection associated with a systemic inflammatory response.

\subsection{Statistical Analysis}

The patients' characteristics were added to the Microsoft Excel sheet. Descriptive analysis was done using Microsoft Excel 2010. Qualitative data were reported as fre- quency and percentage, whereas quantitative data were reported as a mean and slandered deviation.

\section{Results}

The patient's age range was 8 to 78 years (average 57.3 \pm 11.8 years). Pediatrics patients' (7 cases) age group was 8 to 15 years. Of 210 PCNL surgeries, 134 and 76 cases were carried out in males and females, respectively. Stone characteristics were as follows: 71 pelvic or calyceal solitary stones; 62 non-complete staghorn stones; 17 ureteral stones; 32 renal + ureteral stones (simultaneous renal and ureteral stones); and 28 complete staghorn stones. Average stone size was $3.5 \pm 2.8 \mathrm{~cm}$ (range $0.7 \mathrm{~cm}$ to $11.8 \mathrm{~cm}$ ) (Table 1).

Regarding operation time, the mean operation length was $85.0 \pm 29.4 \mathrm{~min}(64.7 \mathrm{~min}, 73.8 \mathrm{~min}, 78.0 \mathrm{~min}$, $88.2 \mathrm{~min}, 108.5 \mathrm{~min}$ for pelvic or calyceal solitary stones, non-complete staghorn kidney stones, ureteral, renal + ureteral, and complete staghorn stones, respectively (Table 1).

All patients had a hemoglobin level of more than $9 \mathrm{~g}$ before the operation. Only 3 patients $(1.4 \%)$ required postoperative transfusion, which was observed in cases with staghorn stone and multiple tracts. No patient underwent surgical exploration and nephrectomy due to severe postoperative hemorrhage. Two patients needed angiographic embolization due to delayed hemorrhage.

We did not face any pulmonary complications, which may be due to our method because we used lower and middle calyx tracts until it was impossible to remove the stone from the upper calyx. Even in this case, we first tried to push stone from upper calyx to pelvis with water pressure through a puncture needle or Alken cannula. A success rate of $90 \%$ was achieved, and there was no need for upper tract formation. We discharged patients within 24 hours based on the study protocol and using antibiotics, and a followup was considered after 5 days. The mean hospital stay was $21.7 \pm 3.4$ hours. Out of 210 patients, 6 patients had longer stay due to high-grade fever and 3 patients due to severe pain, and also 7 patients refused discharge due to personal or social reasons. Our ambulatory PCNL rate was $97 \%$ (194 out of 210). Also, 19 cases (9.02\%) had a fever; 8 patients had a fever in the evening of the day of surgery, which subsided in the morning and they were discharged, but 6 patients had longer stay and fever lasting $2-3$ days, and 5 patients readmitted due to fever.

Within 72 hours, 5 patients were readmitted due to high-grade fever, 3 patients due to haematuria, 4 patients due to pain and dysuria, and all these patients were discharged after 2 - 4 days with conservative treatment. Thus, our readmission rate was $6.18 \%$ (12 readmitted out of 194). No sepsis and mortality were noted. Minor soakage was 


\begin{tabular}{|c|c|}
\hline Variables & Values $^{a}$ \\
\hline \multicolumn{2}{|l|}{ Stone characteristics } \\
\hline Complete staghorn stones & $28(13.33)$ \\
\hline Kidney + ureteral stones & $32(15.23)$ \\
\hline Pelvic or calyceal solitary stones & $71(33.80)$ \\
\hline Non-complete staghorn stones & $62(29.52)$ \\
\hline Ureteral stones & $17(8.09)$ \\
\hline \multicolumn{2}{|l|}{ Operating time (min) } \\
\hline Pelvic or calyceal solitary stones & $64.7 \pm 17.3$ \\
\hline Non-complete staghorn kidney stones & $73.8 \pm 21.8$ \\
\hline Ureter & $78.0 \pm 24.1$ \\
\hline Kidney + ureteral stones & $88.2 \pm 31.4$ \\
\hline Overall average operation time & $85.0 \pm 29.4$ \\
\hline Complete staghorn stones & $108.5 \pm 42.7$ \\
\hline Average hospital stay & $21.7 \pm 3.4$ \\
\hline \multicolumn{2}{|l|}{ Visual analogue scale } \\
\hline Zero post-operative day & $3.86 \pm 0.9$ \\
\hline First post-operative day & $1.7 \pm 0.6$ \\
\hline Discharge after $24 h(n=210)$ & $194(97)$ \\
\hline Readmission $(\mathbf{n}=194)$ & $12(6.18)$ \\
\hline \multicolumn{2}{|l|}{ Complications } \\
\hline Post-operative fever & $19(9.02)$ \\
\hline Transfusion rate & $3(1.40)$ \\
\hline Angioembolisation rate & $2(0.95)$ \\
\hline Pulmonary complications & - \\
\hline Re-exploration or nephrectomy & - \\
\hline Major leak & - \\
\hline Sepsis & - \\
\hline Mortality & - \\
\hline
\end{tabular}

${ }^{\text {a }}$ Values are expressed as No. (\%) or mean \pm SD

noted at the tract site, and on days 1 and 5, the wound was healed. No major leak was noted (Table 1).

\section{Discussion}

We aimed at evaluating the applicability and feasibility of ambulatory PCNL. The average duration of surgery was $85.0 \pm 29.4 \mathrm{~min}$, which was comparable with that reported by Rana et al. (7) ( $83.5 \mathrm{~min}$ ), but Al-Ba'adani et al. (8) reported an average of $46.3 \mathrm{~min}$. Singh et al. (9) carried out (10) ambulatory PCNL with an average operation duration of $48.4 \mathrm{~min}$, and Alyami et al. (10) reported $45.9 \mathrm{~min}$ being shorter than the mean length revealed by Shahrour et al. (11) (65 min) and Tefeki et al. (12) (59.6 min). Differences in studies may be due to the used method to calculate the operation time and burden of the stones. Al-Ba'adani et al. (8) determined the operation time from puncturing the pelvic calyceal system until the end of the operation; however, Shahrour et al. (11) and Rana et al. (7) determined that from the beginning of the surgery to the end of the operation. We calculated the procedure time from cystoscopy, and we faced a large stone burden; therefore, our operation time was longer.

No significant intraoperative complication was reported, and the tubeless method was applied for patients in a prone position by placing a double J-stent in an antegrade fashion at the end of the surgery.

Haemostatic agents can be applied as an adjuvant in tubeless PCNL for minimizing or eliminating the bleeding or extravasations risk following tubeless PCNL (13). In Sofer et al. (14) study, 392 cases were subjected to tubeless PCNL without adjuvant hemostatic agents, and tracts were sealed with a parietal suture and formed a closed retroperitoneal compartment. No hemostatic agent was used in our research for sealing the track. Many researchers, such as Tefekli et al. (12) and by Shahrour et al. (11) closed the tracts with sutures. We did not even suture the track and only used hand compression for $5 \mathrm{~min}$.

Regarding post-operative blood transfusion, only 3 patients $(1.4 \%)$ required post-operative transfusion, which was used for staghorn stones and in multiple tracts. Other studies reported a rate of post-operative transfusion as follows: $4 \%$ by Rana et al. (7) and $4.13 \%$ by Al-Ba'adani et al. (8), $10 \%$ by Shoma et al. (15), and $2.02 \%$ in Giusti et al. (16).

Considering the complications of PCNL and the needed preventive and control measures, Seitz et al. (17) reported the prevalence of fever after the PCNL to be 2.8 - 32.1\% caused by several factors, like the operation duration, the used irrigation fluid amounts, the stone size (> $20 \mathrm{~mm}$ ), bacterial load in urine, the obstruction intensity, and the existence of bacteria in the stones. We found the post-operative fever rate of $9.02 \%$ that was 30\% lower than the rate announced by $\mathrm{Ni}$ et al. (18) and over the rates revealed by Al-Ba'adani et al. (8) (3.3\%) and Shah et al. (13) (6.2\%).

Based on Rana et al. (7) report, post-operative urinary leakage was not reported in 104 patients undergoing tubeless supine PCNL. The same findings were reported by Giusti et al. (16), who studies 99 patients subjected to tubeless PCNL with placing double J-stent in an antegrade manner at the end of the surgery, similar to our patients. In our research, no major leakage was found, and a minor leak could be resolved spontaneously within 24 hours after the surgery that was less than the rate (4\%) of post-operative 
leakage reported by Shoma et al. (15).

We used a stone-free rate of $100 \%$ as it was our inclusion criteria. We also applied VAS to measure the mean pain score that was obtained $3.86 \pm 0.86$ on the day of surgery and $1.7 \pm 0.64$ on the first day after surgery. These values are comparable to those declared by Shoma et al. (15) $(3.2$ \pm 1.8 and $1.6 \pm 1.9$, respectively); however, they seem less than the values reported by Giusti et al. (16) (3.5) on the first day after surgery and Singh et al. (9) (2.4). In addition, analgesics were less needed compared with conventional PCNL, as PNT gives more discomfort and pain, and the average analgesic requirement was $1 \mathrm{mg} / \mathrm{kg}$ diclofenac.

Also, 194 patients were sent home within 24 hours of PCNL after ensuring appropriate pain management as well as proper family support, whereas, for those who were not discharged after surgery (non-ambulatory), appropriate management was done. Our ambulatory PCNL rate was $97 \%$ (194 out of 210 ) and the readmission rate was $6.18 \%$ (12 readmitted out of 194).

We found an average hospital stay of $21.7 \pm 3.4$ hours, whereas Shah et al. (13) declared 34 hours, Tefekli et al. (12) 39 hours, Singh et al. (9) 40 hours, Alyami et al. (10) 41 hours, but it was shorter in a study by Shehrour et al. (11) (4 h) owing to their severe standards, and longer in Al' Badani et al. (8) (50.7 h) and Shoma et al. (15) (65 $\pm 49 \mathrm{~h})$ studies that were based on their report according to which, a tubeless procedure using external ureteral drainage prolongs the hospital stay one day more in comparisons with internal double J-stenting. Considering the hospital stay duration, $97 \%$ of our cases were able to be safely discharged 24 hours after surgery. According to Alyami et al. (10) study, $66 \%$ of their cases were able to be discharged following an overnight stay, and they suggested that an overnight hospital stay following PCNL represents a good strategy for improved bed use in selected cases that can also decrease hospital cost for post-operative care.

Our study included simple to complex stones, and we considered post-operative fever, transfusion rate, angioembolisation rate, pulmonary complications, VAS, reexploration, the need for nephrectomy, major leak, sepsis, and mortality. All these factors were comparable to other studies having conventional PCNL. None of these complications and also the complexity of stones can affect the early discharge of patients. In our study, we only considered tubeless PCNL that may be the reason for less pain, comfort, and psychological well-being. Patients having PNT should also be studied.

\subsection{Conclusion}

Ambulatory (daycare) PCNL procedure is an applicable and feasible procedure under selected criteria; however, more investigations using a larger sample size are needed.

\section{Footnotes}

Authors' Contribution: Rehan Fareed developed the original idea and the protocol, abstracted and analyzed data, wrote the manuscript, and is a guarantor. Huma Shamim contributed to the development of the protocol, abstracted the data, and prepared the manuscript.

Conflict of Interests: None to declare.

Funding/Support: None to declare.

\section{References}

1. Fernstrom I, Johansson B. Percutaneous pyelolithotomy. A new extraction technique. Scand J Urol Nephrol. 1976;10(3):257-9. doi: 10.1080/21681805.1976.11882084. [PubMed: 1006190].

2. Tailly T, Denstedt J. Innovations in percutaneous nephrolithotomy. Int J Surg. 2016;36(Pt D):665-72. doi: 10.1016/j.ijsu.2016.11.007. [PubMed: 27826048].

3. Patel SR, Nakada SY. The modern history and evolution of percutaneous nephrolithotomy. J Endourol. 2015;29(2):153-7. doi: 10.1089/end.2014.0287. [PubMed: 25093997].

4. Kamal W, Kallidonis P, Kyriazis I, Liatsikos E. Minituriazed percutaneous nephrolithotomy: What does it mean? Urolithiasis. 2016;44(3):195-201. doi: 10.1007/s00240-016-0881-x. [PubMed: 27084193].

5. Proietti S, Giusti G, Desai M, Ganpule AP. A critical review of miniaturised percutaneous nephrolithotomy: Is smaller better? Eur Urol Focus. 2017;3(1):56-61. doi:10.1016/j.euf.2017.05.001. [PubMed:28720368].

6. Ghosh A, Oliver R, Way C, White L, Somani BK. Results of day-case ureterorenoscopy (DC-URS) for stone disease: Prospective outcomes over 4.5 years. World J Urol. 2017;35(11):1757-64. doi: 10.1007/s00345-0172061-1. [PubMed: 28620694]. [PubMed Central: PMC5649591].

7. Rana AM, Bhojwani JP, Junejo NN, Das Bhagia S. Tubeless PCNL with patient in supine position: Procedure for all seasons?With comprehensive technique. Urology. 2008;71(4):581-5. doi: 10.1016/j.urology.2007.10.059. [PubMed: 18279935].

8. Al-Ba'adani TH, Al-Kohlany KM, Al-Adimi A, Al-Towaity M, Al-Baadani T, Alwan M, et al. Tubeless percutaneous neprolithotomy: The new gold standard. Int Urol Nephrol. 2008;40(3):603-8. doi: 10.1007/s11255-0079305-8. [PubMed: 18092142].

9. Singh I, Kumar A, Kumar P. "Ambulatory PCNL" (tubeless PCNL under regional anesthesia) - a preliminary report of 10 cases. Int Urol Nephrol.2005;37(1):35-7.doi:10.1007/s11255-004-6706-9. [PubMed: 16132756].

10. Alyami F, Norman RW. Is an overnight stay after percutaneous nephrolithotomy safe? Arab J Urol. 2012;10(4):367-71. doi: 10.1016/j.aju.2012.07.006. [PubMed: 26558051]. [PubMed Central: PMC4442911].

11. Shahrour W, Andonian S. Ambulatory percutaneous nephrolithotomy: Initial series. Urology. 2010;76(6):1288-92. doi: 10.1016/j.urology.2010.08.001. [PubMed: 21130245].

12. Tefekli A, Altunrende F, Tepeler K, Tas A, Aydin S, Muslumanoglu AY. Tubeless percutaneous nephrolithotomy in selected patients: A prospective randomized comparison. Int Urol Nephrol. 2007;39(1):5763. doi: 10.1007/s11255-006-9040-6. [PubMed: 17171416].

13. Shah HN, Hegde S, Shah JN, Mohile PD, Yuvaraja TB, Bansal MB A prospective, randomized trial evaluating the safety and efficacy of fibrin sealant in tubeless percutaneous nephrolithotomy. J Urol. 2006;176(6 Pt 1):2488-93. doi: 10.1016/j.juro.2006.07.148. [PubMed: 17085137]. 
14. Sofer M, Beri A, Friedman A, Aviram G, Mabjeesh NJ, Chen J, et al. Extending the application of tubeless percutaneous nephrolithotomy. Urology. 2007;70(3):412-7. doi: 10.1016/j.urology.2007.03.082. [PubMed: 17905084].

15. Shoma AM, Elshal AM. Nephrostomy tube placement after percutaneous nephrolithotomy: Critical evaluation through a prospective randomized study. Urology. 2012;79(4):771-6. doi: 10.1016/j.urology.2011.09.042. [PubMed: 22130359].

16. Giusti G, Piccinelli A, Maugeri O, Benetti A, Taverna G, Graziotti P. Percutaneous nephrolithotomy: tubeless or not tubeless? Urol Res. 2009;37(3):153-8. doi: 10.1007/s00240-009-0183-7. [PubMed:
19326109].

17. Seitz C, Desai M, Hacker A, Hakenberg OW, Liatsikos E, Nagele U, et al. Incidence, prevention, and management of complications following percutaneous nephrolitholapaxy. Eur Urol. 2012;61(1):146-58. doi: 10.1016/j.eururo.2011.09.016. [PubMed: 21978422].

18. Ni S, Qiyin C, Tao W, Liu L, Jiang $\mathrm{H}, \mathrm{Hu} \mathrm{H}$, et al. Tubeless percutaneous nephrolithotomy is associated with less pain and shorter hospitalization compared with standard or small bore drainage: a metaanalysis of randomized, controlled trials. Urology. 2011;77(6):1293-8. doi: 10.1016/j.urology.2010.10.023. [PubMed: 21256562]. 